\title{
Hepatoid adenocarcinoma of the stomach: A case report of a rare type of gastric cancer
}

\author{
ZHIHONG SHEN, XIBO LIU, BAOCHUN LU and MINFENG YE \\ Department of General Surgery, Shaoxing People's Hospital, Shaoxing Hospital of Zhejiang University, \\ Shaoxing, Zhejiang 312000, P.R. China
}

Received November 14, 2014; Accepted October 9, 2015

DOI: $10.3892 / \mathrm{ol} .2015 .4023$

\begin{abstract}
Hepatoid adenocarcinoma of the stomach (HAS) is a rare type of gastric cancer with an extremely poor prognosis. The current study reports a rare case of HAS, characterized by gastric cancer and infiltration of cancer cells into the left liver lobe, as well as lymphadenectasis. The expression of $\alpha$-fetoprotein (AFP) was markedly increased in the tumor cells of the liver neoplasms. A gastric biopsy indicated highly, moderately and poorly differentiated papillary adenocarcinoma. The patient underwent two cycles of chemotherapy with oxaliplatin (130 mg; day 1) and capecitabine (2 mg, twice daily; days 1-14). At 7 weeks after the chemotherapy, an expanded gastrectomy and radical resection of left lung lobe were performed on the operable lesion. AFP expression was significantly decreased following the procedure. A literature review was also conducted by searching PubMed/Medline, indicating that surgery and chemotherapy may positively affect the outcomes of HAS patients.
\end{abstract}

\section{Introduction}

Hepatoid adenocarcinoma is an extrahepatic tumor with an incidence of $0.38-0.73 \%$ (1). The occurrence of this malignancy has been described in several organs, including the lungs, gallbladder, esophagus, uterus and stomach (2). Hepatoid adenocarcinoma of stomach (HAS) refers to a rare type of gastric carcinoma characterized by a distinct morphology and elevated $\alpha$-fetoprotein (AFP) levels (3). The diagnosis of HAS is largely depend on the pathological analysis. In 1981, Kodama et al (4) initially described two histologic types of AFP-producing gastric carcinoma with medullary or papillotubular arrangements. Subsequently, Ishikura et al (5)

Correspondence to: Professor Xibo Liu, Department of General Surgery, Shaoxing People's Hospital, Shaoxing Hospital of Zhejiang University, 568 Zhongxing North Road, Shaoxing, Zhejiang 312000, P.R. China

E-mail: shensx0531@126.com

Key words: hepatoid adenocarcinoma of stomach, gastric cancer, $\alpha$-fetoprotein, chemotherapy, prognosis proposed the term 'hepatoid adenocarcinoma of the stomach' for primary gastric carcinomas characterized by hepatoid differentiation and the production of large amounts of AFP. At present, the prognosis of HAS is rather poor and according to a literature search of PubMed/Medline between January 2001 and December 2013, only a few cases of this disease have been reported (Table I) (6-8). The current study reports a case of HAS, and summarizes the treatment and outcome for the disease.

\section{Case report}

A 70-year-old male patient presented to Shaoxing People's Hospital (Shaoxing, China) due to muscle weakness and palpitations lasting for 2 months. No abnormalities were noted during the physical examination. During laboratory tests, fecal occult blood was noted, and the patient's AFP level was $14,399.9 \mathrm{ng} / \mathrm{ml}$ (normal range, 0-13.4 ng/ml). Ultrasound examination indicated an occupying lesion in right upper quadrant. Computed tomography imaging was performed, revealing gastric cancer and infiltration of cancer cells into the left lobe of the liver, as well as lymphadenectasis of the group 1 and 3 nodes (Fig. 1A). Gastroscopy and pathological tests revealed irregular bulges in the gastric antrum and oedema in the peripheral mucous membrane. In addition, highly-differentiated adenocarcinoma cells were observed. On this basis, the patient was diagnosed with adenocarcinoma of the gastric antrum (Figs. 1B and 2A). The diagnosis of HAS was primarily based on the presence of the following aspects: papillary adenocarcinoma of high, moderate or low grade; mucinous adenocarcinoma; undifferentiated carcinoma and poorly differentiated hepatocellular carcinoma combined with presence of hyaline bodies; poorly differentiated hepatocellular carcinoma and hyaline body-like structures in the liver cancer specimen. Two cycles of chemotherapy with oxaliplatin (130 mg; day 1) and capecitabine (2 mg, twice daily; days 1-14) were administered.

At 7 weeks after chemotherapy, resection of the stomach and external lobe of the left liver were conducted, during which a tumor mass measuring $\sim 2.0 \times 1.5 \times 1.0 \mathrm{~cm}$ was observed in gastric corpus. Meanwhile, a gastric biopsy revealed an ulcerating tumor $(4.0 \times 3.0 \times 1.5 \mathrm{~cm})$ from the gastric angle to the gastric antrum, and infiltration of cancer cells into left liver $(4.0 \times 3.0 \times 3.0 \mathrm{~cm})$. No postoperative complications were 
A

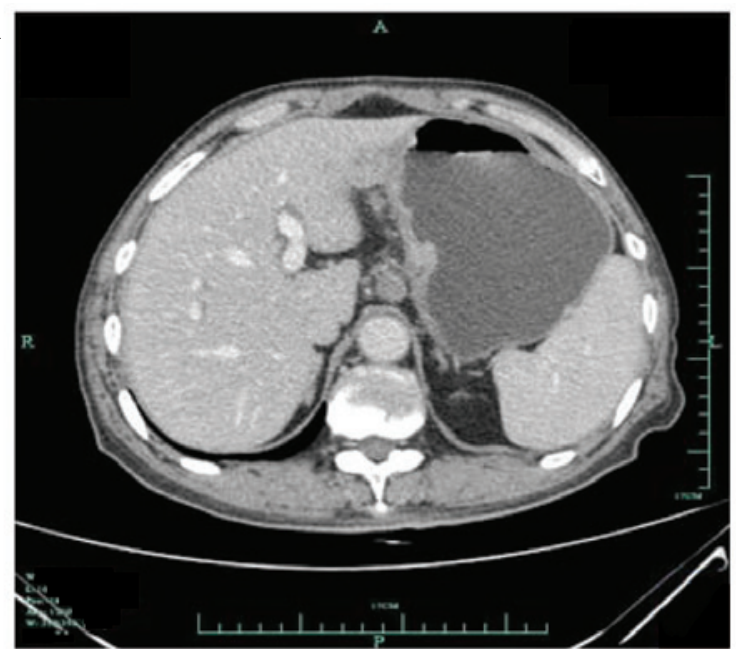

B

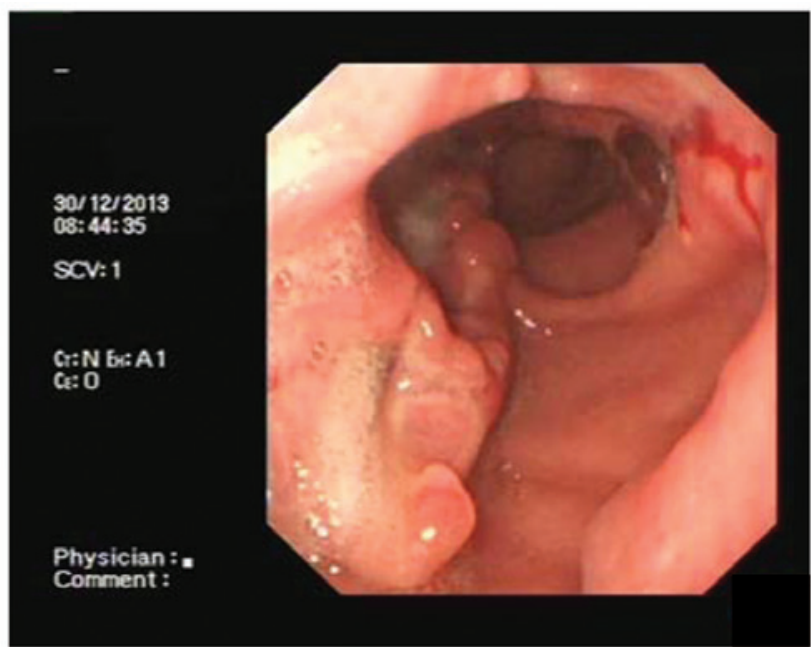

Figure 1. Imaging results. (A) Computed tomography imaging revealed a lesion in the left lobe of the liver prior to surgery. (B) Endoscopy identified a protruding lesion in the gastric antrum prior to chemotherapy.
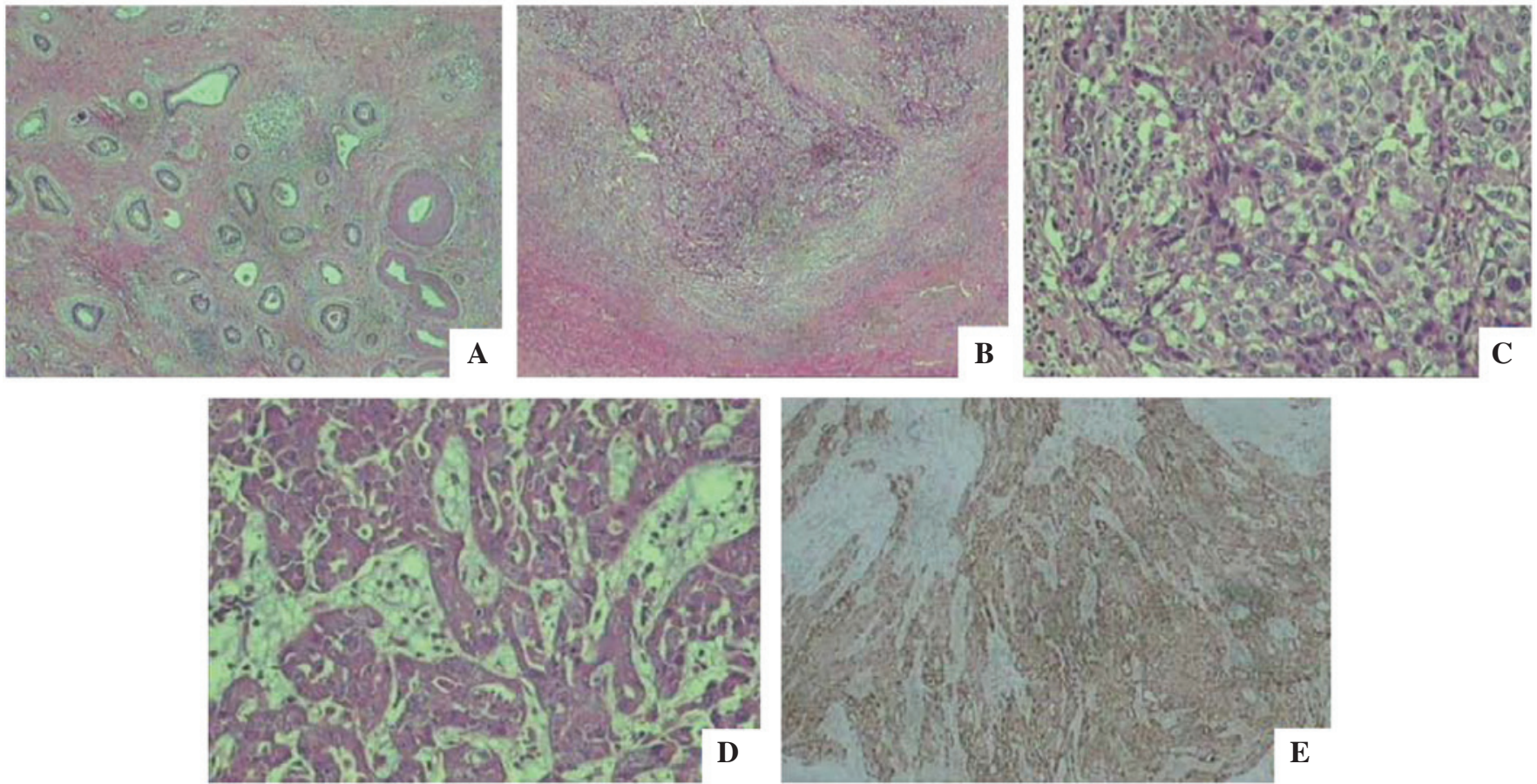

Figure 2. Histochemical and immunostaining results. (A) Highly differentiated adenocarcinoma and small cellular atypia was identified in gastric samples (H\&E staining; magnification, x100). (B) Poorly differentiated adenocarcinoma was identified in hepatic samples; the tumor demonstrated an infiltrative growth pattern (H\&E staining; magnification, x100). (C) Poorly, moderately and highly differentiated hepatocellular carcinomas were identified in the liver (H\&E staining; magnification, x200). (D) Poorly differentiated adenocarcinoma was identified in the gastric sample, which exhibited hepatocyte-like differentiation and interstitial edema (H\&E staining; magnification, x200). (E) $\alpha$-fetoprotein expression in hepatic samples (magnification, x100). H\&E, hematoxylin and eosin.

reported. Biopsy of liver indicated adenoid carcinoma with no infiltration of cancer cells in the incisal margins, and no lymph node metastasis (Fig. 2B and C). The immunohistochemistry results were $\operatorname{AFP}(+)$, cytokeratin (CK) 19(+), CK7(-) and CK20(-). Biopsy of gastric samples indicated adenocarcinoma (Fig. 2D); the immunohistochemistry results were AFP(-), c-erbB-2(+), E-cadherin(+), epidermal growth factor receptor(+), Ki-67 (+65\%), CK7(+) and CK20(+). AFP levels were measured on postsurgical days 9 and 30 at 116.7 and $17.3 \mathrm{ng} / \mathrm{ml}$, respectively. Subsequently, chemotherapy was performed 3 weeks after the after surgery. The chemptherapy regimen, was $130 \mathrm{mg}$ oxaliplatin on day 1 plus $2 \mathrm{mg}$ capecitabine, twice daily, on days 1-14, for two cycles. The patient survived during 7 months of follow-up, at which point the AFP levels were $10.4 \mathrm{ng} / \mathrm{ml}$.

\section{Discussion}

For patients with HAS, the initial symptoms are usually upper abdominal pain (9); more rarely, individuals may also exhibit melena. On pathological evaluation, elevated AFP is commonly noted in these patients. In such cases, the AFP is 
Table I. Literature review from January 2001 to December $2013^{\mathrm{a}}$.

\begin{tabular}{|c|c|c|c|c|c|}
\hline Author (reference no.) & Gender & Age (years) & AFP & Treatment & Follow-up \\
\hline Gálvez-Muñoz et al (7) & Male & 75 & Positive & $\begin{array}{l}\text { Palliative total gastrectomy; palliative } \\
\text { chemotherapy with cisplatin and } \\
\text { capecitabine ( } 6 \text { cycles) }\end{array}$ & $\begin{array}{l}\text { Alive at } \\
8 \text { months } \\
\text { post surgery }\end{array}$ \\
\hline Ahn et al (6) & Male & 68 & Positive & $\begin{array}{l}\text { Billroth type II subtotal gastrectomy; } \\
\text { palliative chemotherapy with cisplatin } \\
\text { and capecitabine; second-line palliative } \\
\text { chemotherapy with fluororacil, } \\
\text { leucovorin and irinotecan }\end{array}$ & $\begin{array}{l}\text { Alive during } \\
21 \text { months } \\
\text { of follow-up }\end{array}$ \\
\hline \multirow[t]{3}{*}{ Ye et al (8) } & Male & 58 & Positive & $\begin{array}{l}\text { Distal gastrectomy; epirubicin, } \\
\text { oxaliplatin and fluorouracil ( } 6 \text { cycles })\end{array}$ & $\begin{array}{l}\text { Alive at } \\
20 \text { months } \\
\text { post surgery }\end{array}$ \\
\hline & Male & 54 & Positive & $\begin{array}{l}\text { Total gastrectomy with lymph node } \\
\text { dissection; oxaliplatin plus fluorouracil } \\
\text { adjuvant chemotherapy ( } 6 \text { cycles); } \\
\text { paclitaxel and capecitabine ( } 2 \text { cycles })\end{array}$ & $\begin{array}{l}\text { Deceased at } \\
18 \text { months } \\
\text { post surgery }\end{array}$ \\
\hline & Female & 61 & Positive & Chemotherapy with oxaliplatin plus S-1 & $\begin{array}{l}\text { Deceased at } \\
8 \text { months } \\
\text { after treatment }\end{array}$ \\
\hline Present study & Male & 70 & Positive & $\begin{array}{l}\text { Expanded gastrectomy and radical } \\
\text { resection of left lung lobe; chemotherapy } \\
\text { using oxaliplatin and capecitabine }\end{array}$ & $\begin{array}{l}\text { Alive during } \\
7 \text { months of } \\
\text { follow-up }\end{array}$ \\
\hline
\end{tabular}

aSearch of Pubmed/Medline for English language articles using terms 'hepatoid adenocarcinomas of stomach', 'AFP-producing tumor' and 'AFP-producing gastric cancer'. AFP, $\alpha$-fetoprotein.

typically significantly reduced compared with the baseline levels following surgery and chemotherapy.

The diagnosis of HAS is largely dependent upon hematoxylin and eosin (H\&E) staining and immunohistochemical staining. When stained with H\&E, HAS typically exhibits similar features to hepatocellular carcinoma. In addition, proliferation of polygonal tumor cells is observed in trabecular and intestinal-like structures. For the differential diagnosis, immunohistochemical staining is required. In the current case, AFP expression was confirmed using immunohistochemical staining, as shown in Fig. 2E.

All articles cited in the Medline/PubMed database between January 2001 and December 2013 were searched using the terms 'hepatoid adenocarcinomas of stomach', 'AFP-producing tumor' and 'AFP-producing gastric cancer'. Simultaneously, a manual search of all relevant articles was performed. The search was limited to English language articles. A total of 6 patients (including the current case) were included in the literature review (Table I) (6-8).

To date, no consensus has been reached regarding the treatment of HAS, as little data are available in the literature $(10,11)$. Usually, the disease is treated using similar strategies as those used to treat gastric adenocarcinoma. Radical surgery is considered to be the optimal treatment option and, at the same time, adjuvant chemotherapy and radiotherapy should be performed according to the indications of the gastric cancer (12). In the literature review, 4 patients $(66.7 \%)$ received gastrectomy and chemotherapy, while 2 patients $(33.3 \%)$ received only chemotherapy. For patients with metastasis, including metastasis to the liver, simultaneous resections are necessary (2). In the present case, the patient received oxaliplatin (130 $\mathrm{mg}$; day 1$)$ and capecitabine (2 mg, twice daily; days 1-14), and radical surgery was performed to remove the lesions.

The literature review indicates that men are more prone to developing HAS compared with women. In addition, the mean age of the patients was 64.3 years, demonstrating that individuals of advanced age are more prone to developing the disease. The rate of positive AFP expression was $100.0 \%$. Following surgery, AFP expression was also used as a marker for the subsequent treatment. In the present study, AFP expression levels were significantly reduced on postoperative day 30 compared with that on day 9. In addition, AFP expression levels were stable during the 7-month follow-up.

In summary, HAS is a rare type of carcinoma with poor prognosis. The current study presented a case of HAS occurring in a 70-year-old male, and summarized the treatment outcomes of the chemotherapy and/or gastrectomy in previous studies of this disease. The present results indicated surgery and chemotherapy may positively affect the outcome of similar patients. As the number of cases is still limited, further randomized controlled trials are necessary to confirm the efficacy of this treatment for HAS.

\section{References}

1. Gao YB, Zhang DF, Jin XL and Xiao JC: Preliminary study on the clinical and pathological relevance of gastric hepatoid adenocarcinoma. J Dig Dis 8: 23-28, 2007. 
2. Baek SK, Han SW, Oh DY, Im SA, Kim TY and Bang YJ Clinicopathologic characteristics and treatment outcomes of hepatoid adenocarcinoma of the stomach, a rare but unique subtype of gastric cancer. BMC Gastroenterol 11: 56, 2011.

3. Gitlin D, Perricelli A and Gitlin GM: Synthesis of -fetoprotein by liver, yolk sac, and gastrointestinal tract of the human conceptus. Cancer Res 32: 979-982, 1972.

4. Kodama T, Kameya T, Hirota T, Shimosato Y, Ohkura H, Mukojima $\mathrm{T}$ and Kitaoka H: Production of $\alpha$-fetoprotein, normal serum proteins, and human chorionic gonadotropin in stomach cancer: Histologic and inmunohistochemical analysis of 35 cases. Cancer 4: 1647-1655, 1981 .

5. Ishikura H, Fukasawa Y, Ogasawara K, Natori T, Tsukada Y and Aizawa M: An AFP-producing gastric carcinoma with features of hepatic differentiation: A case report. Cancer 56: 840-848, 1985.

6. Ahn JS, Jeon JR, Yoo HS, Park TK, Park CK, Sinn DH and Paik SW: Hepatoid adenocarcinoma of the stomach: An unusual case of elevated alpha-fetoprotein with prior treatment for hepatocellular carcinoma. Clin Mol Hepatol 19: 173-178, 2013.

7. Gálvez-Muñoz E, Gallego-Plazas J, Gonzalez-Orozco V, Menarguez-Pina F, Ruiz-Maciá JA and Morcillo MA: Hepatoid adenocarcinoma of the stomach - a different histology for not so different gastric adenocarcinoma: A case report. Int Semin Surg Oncol 6: 13, 2009.
8. Ye MF, Tao F, Liu F and Sun AJ: Hepatoid adenocarcinoma of the stomach: A report of three cases. World J Gastroenterol 19: 4437-4442, 2013

9. Liu X, Cheng Y, Sheng W, Lu H, Xu X, Xu Y, Long Z, Zhu H and Wang Y: Analysis of clinicopathologic features and prognostic factors in hepatoid adenocarcinoma of the stomach. Am J Surg Pathol 34: 1465-1471, 2010.

10. Inagawa S, Shimazaki J, Hori M, Yoshimi F, Adachi S, Kawamoto T, Fukao K and Itabashi M: Hepatoid adenocarcinoma of the stomach. Gastric Cancer 4: 43-52, 2001.

11. Trompetas V, Varsamidakis N, Frangia K, Polimeropoulos V and Kalokairinos E: Gastric hepatoid adenocarcinoma and familial investigation: Does it always produce alpha-fetoprotein? Eur J Gastroenterol Hepatol 15: 1241-1244, 2003.

12. Xie Y, Zhao Z, Li P, Wang Y, Guo C, Wang X, Tang W, Liu Q, Lu $\mathrm{N}$, Xue L and Zhao D: Hepatoid adenocarcinoma of the stomach is a special and easily misdiagnosed or missed diagnosed subtype of gastric cancer with poor prognosis but curative for patients of pN0/1: The experience of a single center. Int J Clin Exp Med 8: 6762-6772, 2015. 